\title{
COLLATERAL BLOOD FLOW AFTER BRANCH ARTERIOLAR OCCLUSION IN THE HUMAN RETINA* $\dagger$
}

\author{
BY \\ C. T. DOLlERY, D. W. HILL, J. W. PATERSON, P. S. RAMALHO + , \\ AND E. M. KOHNER \\ From the Department of Medicine, Postgraduate Medical School, and the Professorial Unit, \\ Moorfields Eye Hospital, London
}

OCCLUSION of a branch retinal arteriole in the human eye may follow either embolism or thrombosis. Emboli usually originate from the left heart or carotid arteries and thrombosis most often occurs as a complication of hypertension or diabetes. If the vascular occlusion persists, collateral blood flow may be established by tortuous channels linking the blocked vessel with patent vessels nearby. Clinical reports of the occurrence of arteriolar collaterals in the human retina have been published (Jensen, 1938; Wise, 1956; Ballantyne and Michaelson, 1962; Larsen, 1964), but none has established how effective these collaterals are in restoring flow.

In this paper we record for the first time four patients with branch retinal arteriolar occlusion and collateral formation, all of whom were studied from the dynamic standpoint by both colour photography and fluorescence angiography. In three of the four it was possible to estimate the effectiveness of the collaterals in carrying flow to the blocked arteriole by measuring the velocity of the fluorescent dye front in the blood vessels.

\section{Methods}

Colour and fluorescence photographs of the retinae were taken with the Carl Zeiss retinal camera, using methods already published (Dollery, Hodge, and Engel, 1963). The fluorescent dye was injected intravenously in three cases and into the innominate artery in one.

\section{Case Reports}

Case 1, a man aged 51, complained of 7 weeks' altitudinal hemianopia in the lower half of the right visual field.

Examination.-The visual acuity was $6 / 9$ in the right and $6 / 5$ in the left. The right pupillary reflex was reduced. A large arcuate scotoma was seen in the right eye, starting just beyond the disc and extending across to the nasal horizontal raphe. Much of this was absolute.

Both fundi showed Grade III hypertensive changes with narrowed irregular arterioles and retinal haemorrhages. A small plaque was present in the right superior artery; this vessel was not completely occluded, but its branches were unequally filled.

Fluorescence Angiogram.- This revealed that one of the branches of the superior artery did not fill directly from the main stem. Dye passed into this branch retrogradely from the periphery

- Received for publication October 29, 1965.

Address for reprints: Department of Medicine, Royal Postgraduate Medical School, London, W.12.

$\ddagger$ In receipt of a Fellowship from the Gulbenkian Foundation. 
but the individual collateral vessels could not be distinguished (Fig. 1). The velocity of retrograde flow in this vessel was $\mathbf{0 . 2 2} \mathrm{mm}$. per second.

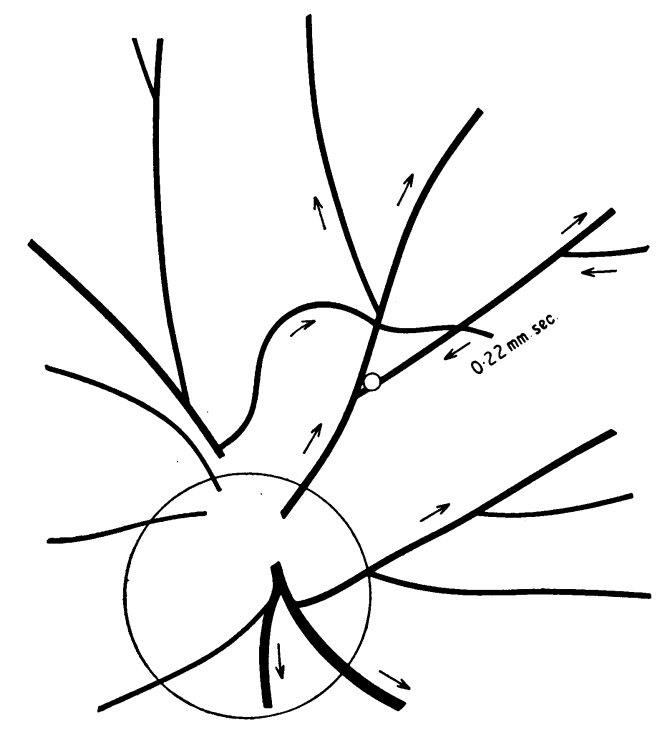

FIG. 1.-Case 1, diagram of right superior nasal area and optic disc. The anatomical arrangement of the main arterioles, the site of the block (- - - ), and retrograde flow in the distal segment of the vessel are shown diagrammatically. An abnormal arteriolar branch arising from the origin of the superior temporal artery crosses the superior nasal vessels but apparently did not feed collateral flow to the blocked superior nasal arteriole.

Case 2, a West Indian housewife aged 40, suffered spontaneous sub-conjunctival haemorrhage 3 weeks before admission to hospital.

Examination.-The fundi showed widespread arteriolar changes with increased light reflex, variability of calibre, and arterio-venous nipping. In the right eye there were, in addition, multiple areas of vascular sheathing, micro-aneurysms, new vessels, and hard exudates. In the superior temporal area a branch of the main artery was completely occluded. The visual acuity was $6 / 6$ in the right eye and $6 / 5$ in the left. There was no significant difference in ophthalmodynamometry readings between the two eyes and no field defect to confrontation.

General Examination.-The blood pressure was $140 / 100 \mathrm{~mm}$. but there were no other abnormal physical signs. A glucose tolerance test was normal and no cause was found for the mild hypertension.

Fluorescence Angiogram.-This was performed by injecting $1 \mathrm{ml}$. of 5 per cent. sodium fluorescein into the innominate artery, and showed many arteriolar collaterals feeding the distal part of the occluded branch arteriole (Figs 2, 3, 4, 5, opposite). The flow in the distal part of the occluded vessel was in the normal direction with a velocity of $1.1 \mathrm{~mm}$. per second.

Case 3, an Irish labourer aged 38, complained of gradual loss of vision in the left eye proceeding to complete blindness in the 3 weeks before admission to hospital.

Examination.-The ophthalmoscope showed oedema of the left optic disc and surrounding retina. There were scattered haemorrhages all over the fundus extending to the periphery. Many of the arteries were sheathed and there were localized narrowed areas near the disc. Two branches of the superior temporal artery were completely occluded distal to the macula. In the right fundus there were haemorrhages and sheathing of the arteries in the temporal periphery.

General Examination.-There was no apparent abnormality but investigation showed that the blood urea varied between 36 and $65 \mathrm{mg}$. $/ 100 \mathrm{ml}$. and the urine contained a trace of protein. An 

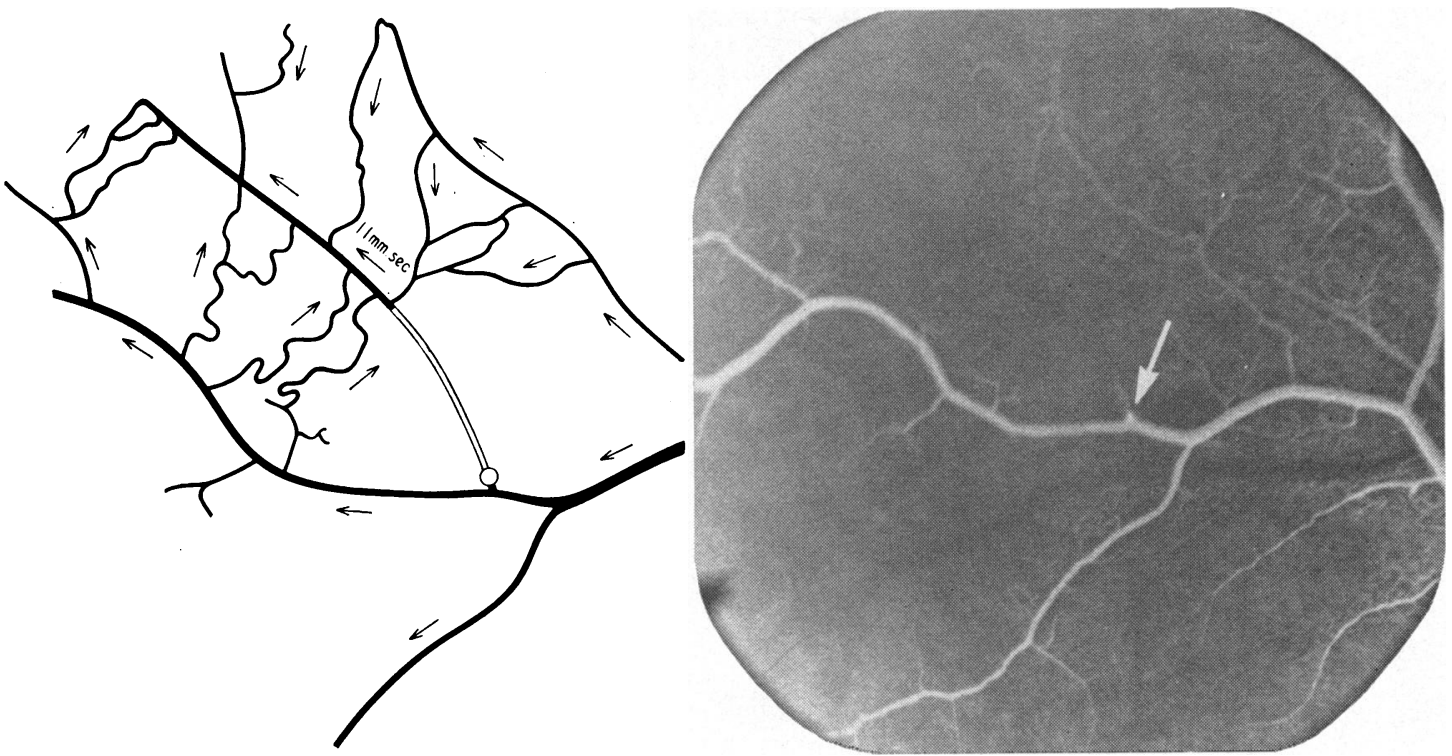

FIG. 2.-Case 2, diagram of main arterioles in peripheral part of territory of right superior temporal arteriole. The site of the block and a length of totally occluded vessel are shown. There were profuse arteriolar collaterals and only the main tributaries of these are shown. Flow in the distal part of the occluded vessel was in the normal

FIG. 3.-Case 2, fluorescence photograph taken $2 \cdot 7$ seconds after injection of $1 \mathrm{ml}$. 5 per cent. sodium fluorescein into the innominate artery. The white lines show fluorescein within the branches of the right superior temporal artery. One branch is occluded at its origin (arrow) and does not fill.

direction at a velocity of $1.1 \mathrm{~mm}$. per second.

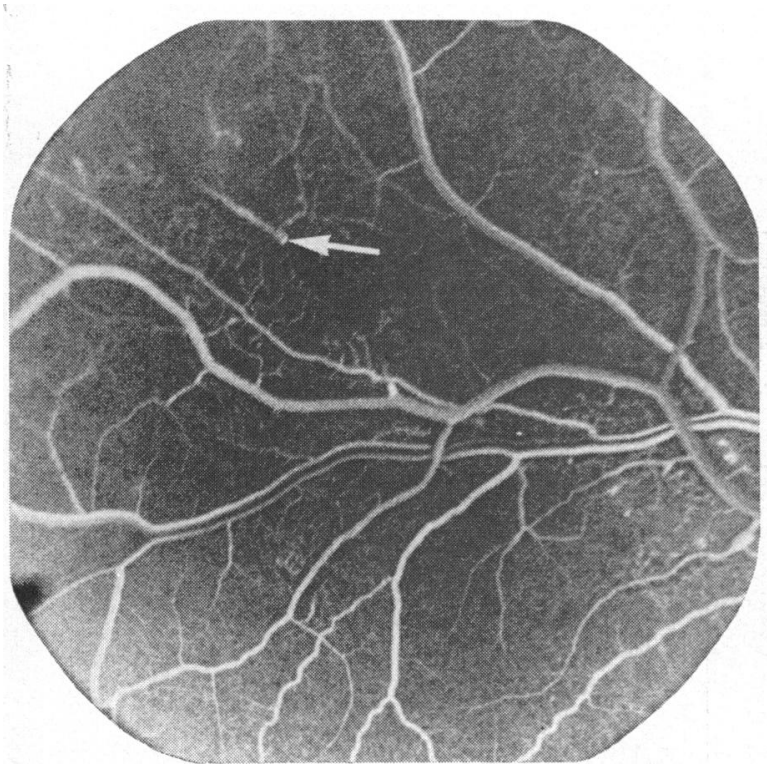

FIG. 4.-Fluorescence photograph taken 1 sec. later than Fig. 3. Numerous collaterals are visible, some of which are already beginning to fill the distal part of the occluded arteriole (arrow).

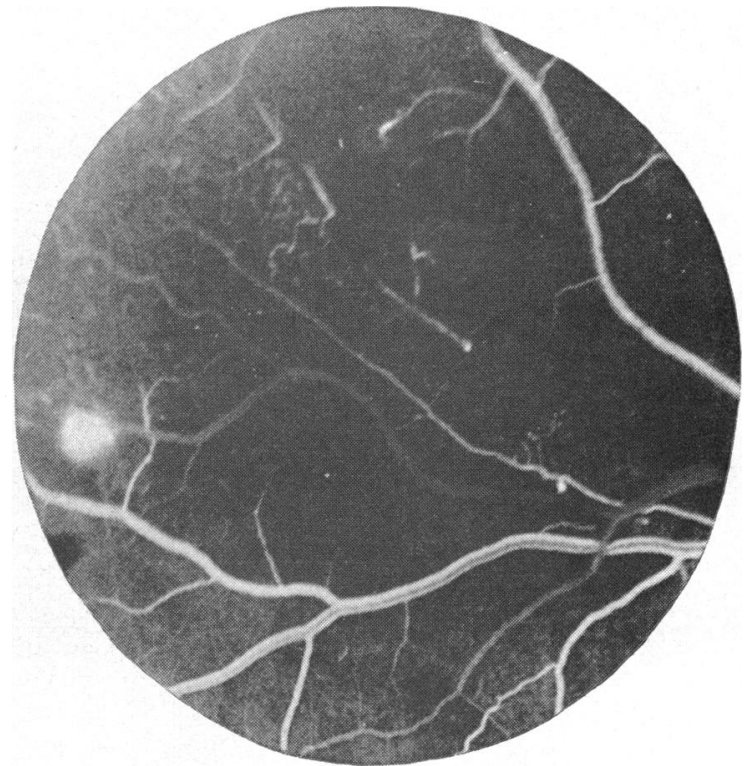

FIG. 5.-Fluorescence photograph taken $1 \mathrm{sec}$. after Fig. 4. A number of separate segments of the occluded arteriole are filling by different collaterals. In the surrounding normal parts of the retina, the dye has left the arterioles and laminar streams are visible within the veins. 
intravenous pyelogram showed the right kidney to be $2 \cdot 2 \mathrm{~cm}$. shorter than the left. However, microscopy and culture of the urine was normal, and the patient was able to concentrate and dilute the urine in a normal fashion. A renal biopsy was normal. Erythrocyte sedimentation rate and serum proteins were normal and a muscle biopsy showed no evidence of arteritis.

Fluorescence Angiogram.-This was performed after the intravenous injection of $5 \mathrm{ml} .5$ per cent. sodium fluorescein. The occluded branch of the superior temporal retinal arteriole did not fill at the same time as the other branches of this vessel. At this stage the territory of the occluded vessel appeared as a dark wedge of non-perfused retina on the angiogram (Ramalho, Dollery, Henkind, Paterson, and Hill, 1965). During the succeeding $16 \mathrm{sec}$. the vessel slowly filled through a large number of peripheral collaterals. The direction of flow in the main occluded arteriole was opposite to normal. The velocity of flow in the main stem of the vessel was $0.26 \mathrm{~mm}$. per second towards the optic disc (Fig. 6).

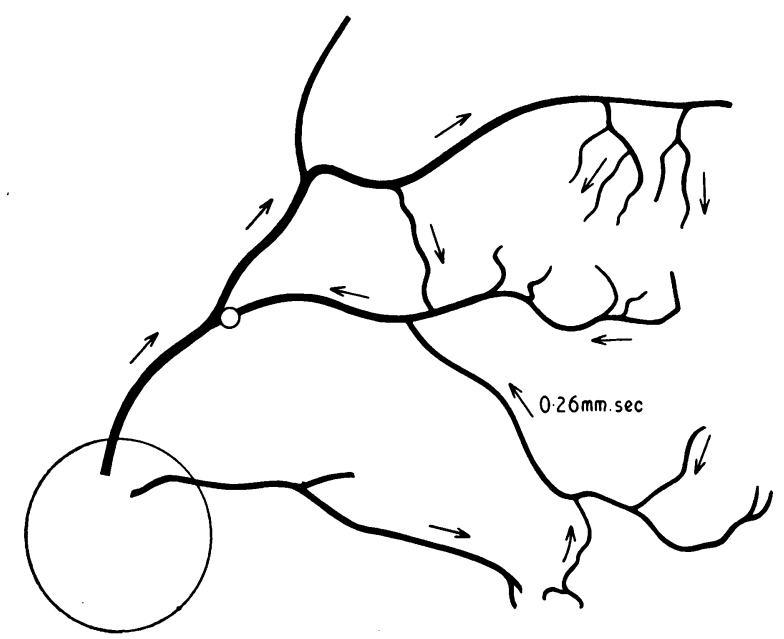

Fig. 6. - Case 3, diagram of main arterioles of left superior temporal circulation in the retina. The site of the block in a main division of the superior temporal arteriole is shown and the arrangement of a number of arteriolar collaterals. It was not possible to trace all of these throughout their length. The collaterals filled the main trunk of the occluded arterioles from the periphery and the direction of flow in it was the reverse of normal. The flow velocity in the main branch was $0.26 \mathrm{~mm}$. per second.

Case 4, an engine-driver aged 52, attended hospital because of transient weakness in the left arm and leg lasting for 3 days.

Examination.-The fundi showed Grade III hypertensive retinopathy with irregular and narrowed arteries, marked arterio-venous nipping, haemorrhages, and soft exudates. The origin of the right inferior nasal artery was occluded on the optic disc. Anastomotic channels were seen with the ophthalmoscope to connect the occluded arteriole with neighbouring normal vessels.

General Examination.-The blood pressure was $270 / 130 \mathrm{~mm}$. with left ventricular hypertrophy. The only clinical evidence of the transient stroke was an increased left knee jerk. An electrocardiogram confirmed the presence of left ventricular hypertrophy. An aortogram outlined atheroma in the right renal artery. Other investigations, including the erythrocyte sedimentation rate, blood urea, urine concentration, urine culture, serum proteins, and electrolytes, were all normal.

Fluorescence Angiogram.-This was carried out by the venous route after the injection of $5 \mathrm{ml}$. 5 per cent. sodium fluorescein. The inferior nasal artery did not fill simultaneously with the other arteries on the optic disc and later filled retrogradely from the periphery. Insufficient pictures were 
taken to establish the velocity of retrograde flow within the distal part of the blocked arteriole. The site of the block and major collaterals visible on colour photographs is shown in Fig. 7 and a diagram of all their connexions in Fig. 8.

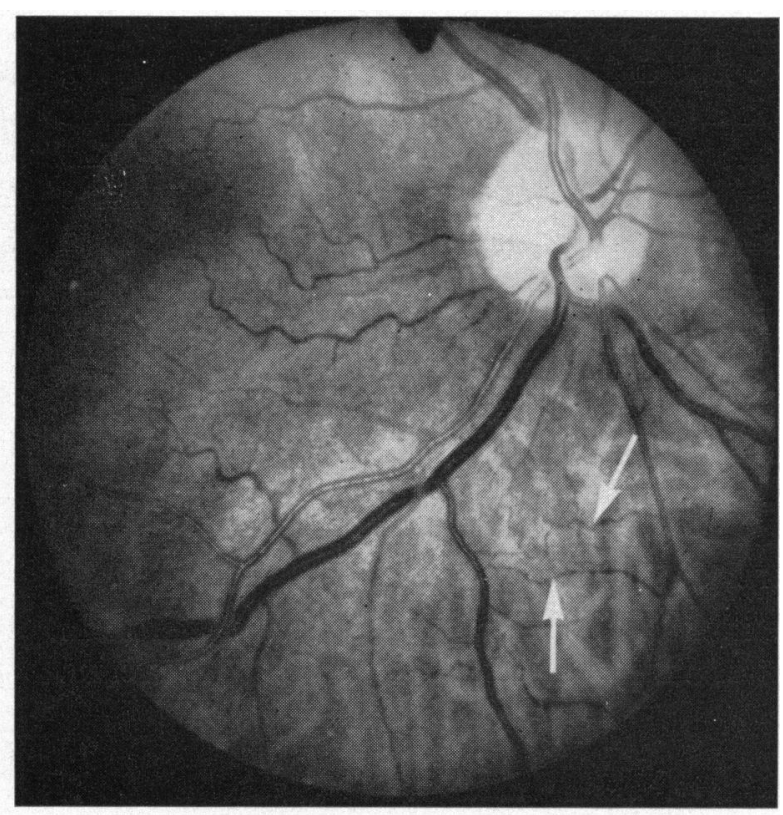

FIG. 7.-Case 4, photograph showing the visible collaterals supplying the inferior nasal artery (arrows). The vessel is blocked at its origin on the disc. Compare with Fig. 8, where all the collaterals are sketched in.

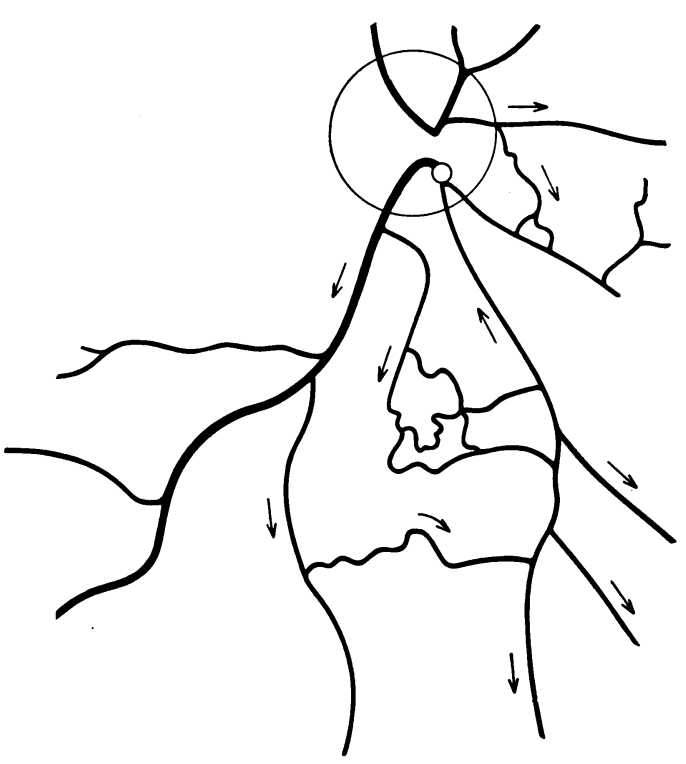

FIG. 8.-Case 4, diagram of main arterioles in right inferior nasal area of retina. The main collaterals are shown. Flow within the occluded arteriole was in the reverse direction to normal but not enough pictures were taken to allow the velocity to be calculated.

\section{Discussion}

The arteriolar occlusions studied in these four patients varied in their aetiology and unfortunately the duration was usually not known. They provide, however, a reasonable guide to the efficacy of arteriolar collaterals in supplying blood to the distal segment of an occluded vessel. The occluded vessels were 50 to 80 microns in diameter and only one supplied the macular area.

The velocity of blood flow in the segment of vessels supplied by collaterals varied from $1 \cdot 1$ to $0.22 \mathrm{~mm}$. per second. In three of the four vessels this flow was in the reverse direction to normal. It may at first sight seem paradoxical that the direction of blood flow within an arteriole can be reversed. In all instances, however, the collateral vessels supplying the arteriole ran in from the periphery of its territory. Thus, blood from neighbouring arterioles passed through these collaterals into the main stem of the occluded vessel and flowed for some way down it in the reverse direction to normal. This blood then passed out through more proximal tributaries through the capillary bed and into branch veins. Thus, the pressure gradient within the arteriole fed by peripheral collaterals was reversed. 
Despite the presence of numerous collaterals, particularly in Case 2 , the velocity of flow in the occluded arteriole was low. It has not yet proved possible to measure the velocity of flow accurately within normal human arterioles, but extrapolation from measurements in the pig retina (which has a similar mean transit time) by cinefluorescence angiography suggests that the normal velocity of flow in arterioles of the size studied in these patients would be between 25 and $50 \mathrm{~mm}$. per second. Thus, the collaterals appeared to be relatively ineffective, but this opinion must be qualified until there is further evidence about the state of the capillary circulation in the territory fed by the obstructed arteriole. Necrosis and atrophy of the nerve fibres and ganglion cell elements supplied by the retinal capillary bed may have occurred in the days following the initial occlusion and so reduced the demand for blood (Kornzweig, Eliasoph, and Feldstein, 1964). In experimental arteriolar occlusion, atrophy of the nerve fibres occurs despite rapid formation of collateral vessels (Dollery, Ramalho, Paterson, Hill, Henkind, Shakib, and Ashton, 1965). By the time the collaterals in the human eye have become fully effective in supplying oxygenated blood to the distal part of the vessel, the demand for oxygen may be much reduced. Furthermore, all four patients had widespread retinal vascular disease which may limit the rate of development of collaterals.

Final judgement concerning the potential effectiveness of arteriolar collaterals in man may have to await the development of a method of keeping the ischaemic retina alive during their development.

\section{Summary}

(1) Four patients with occluded branch retinal arterioles between 50 and 80 microns in diameter were studied by means of colour photography and fluorescence angiography.

(2) All four had collaterals within the retina supplying the distal segment of the occluded vessel.

(3) In three of the four the normal direction of flow within the occluded vessel was reversed.

(4) The velocity of flow within the distal part of the occluded vessel was only $0 \cdot 22$ to $1.1 \mathrm{~mm}$. per second despite the presence of many collaterals. The normal forward flow velocity in a vessel of this calibre is estimated to be between 25 and 50 mm. per second.

(5) One reason for the apparent ineffectiveness of the arteriolar collaterals in providing a substantial flow may be the low demand for blood within that vascular territory owing to necrosis and atrophy of the retinal elements in the hours after vascular occlusion before the collaterals develop.

This work was supported by the Medical Research Council. We are grateful to Mr. P. D. Trevor-Roper at Moorfields Hospital, Dr. M. Harington at the Medical Ophthalmology Unit at Lambeth Hospital, and Dr. Ross Russell at the National Hospital, Queen Square, for referring patients for study. We thank Mrs. Morag Engel, S.R.N., for valuable technical assistance. 


\section{REFERENCES}

Ballantyne, A. J., and Michaelson, I. C. (1962). "Textbook of the Fundus of the Eye", p. 80. Livingstone, Edinburgh.

Dollery, C. T., Hodge, J. V., and Engel, M. (1963). Med. biol. Illus., 13, 4.

-, Ramalho, P. S., Paterson, J. W., Hill, D. W., Henkind, P., Shakib, M., and Ashton, N. (1965). Lancet, 1, 1303.

Jensen, V. A. (1938). Acta ophthal. (Kbh.), 16, 485.

KornzWeig, A. L., Eliasoph, I., and Feldstein, M. (1964). Arch. Ophthal. (Chicago), 71, 542.

LARSEN, H. W. (1964). "Atlas of the Fundus of the Eye", p. 120. Munksgaard, Copenhagen.

Ramalho, P. S., Dollery, C. T., Henkind, P., Paterson, J. W., and Hill, D. W. (1965). Proc. roy. Soc. Med., 58, 914.

WISE, G. N. (1956). Trans. Amer. ophthal. Soc., 54, 729. 